\title{
The Impasse on Overall Survival in Oncology Reimbursement Decision-Making: How Can We Resolve This?
}

\author{
Michael Patrick Lux (1D \\ Oriana Ciani (D) ${ }^{2}$ \\ William CN Dunlop $\mathbb{D}^{3}$ \\ Andrea Ferris ${ }^{4}$ \\ Michael Friedlander 5 \\ 'Department of Gynecology and \\ Obstetrics, Frauenklinik St. Louise \\ Paderborn, St. Josefs-Krankenhaus \\ Salzkotten, Frauen- und Kinderklinik \\ St. Louise Paderborn, Paderborn, \\ Germany; ${ }^{2}$ Centre for Research on \\ Health and Social Care Management, \\ SDA Bocconi, Milan, Italy; ${ }^{3}$ AstraZeneca, \\ City House, Cambridge, UK; ${ }^{4}$ LUNGevity \\ Foundation, Bethesda, MD, USA; ${ }^{5}$ Prince \\ of Wales Clinical School, University of \\ New South Wales and Department of \\ Medical Oncology, The Prince of Wales \\ Hospital, Sydney, NSW, Australia
}

\begin{abstract}
Mature overall survival (OS) data are often unavailable at the time of regulatory and reimbursement decisions for a new cancer treatment. For patients with early-stage cancers treated with potentially curative treatments, demonstrating an OS benefit may take years and may be confounded by subsequent lines of therapy or crossover to the investigational treatment. For patients with advanced-stage cancers, mature OS data may be available but difficult to interpret for similar reasons. There are strong opinions about approval and reimbursement in the absence of mature OS data, with concerns over delay in patient access set against concerns about uncertainty in long-term benefit. This position paper reflects our individual views as patient advocate, clinician or health economist on one aspect of this debate. We look at payer decisions in the absence of mature OS data, considering when and how non-OS trial outcomes could inform decision-making and how uncertainty can be addressed beyond the trial, supporting these views with evidence from the literature. We consider when it is reasonable for payers to expect or not expect mature OS data at the initial reimbursement decision (based on criteria such as cancer stage and treatment efficacy) acknowledging that there are settings in which mature OS data are expected. We propose flexible strategies for generating and appraising patient-relevant evidence, including context-relevant endpoints and quality of life measures, when survival rates are good and mature OS data are not expected. We note that fair reimbursement is important; this means valuing patient benefit as shown through prespecified endpoints and reappraising if there is ongoing uncertainty or failure to show a sustained benefit. We suggest that reimbursement systems continue to evolve to align with scientific advances, because innovation is only meaningful if readily accessible to patients. The proposed strategies have the potential to promote thorough assessment of potential benefit to patients and lead to timely access to effective medicines.
\end{abstract}

Keywords: drug approval, endpoint determination, medical oncology, quality of life, surrogate endpoint, uncertainty
Correspondence: Michael Patrick Lux

Department of Gynecology and

Obstetrics, Frauenklinik St. Louise,

Husener Str. 81, Paderborn, 33098,

Germany

Tel +49525I 864I2I

Emailm.lux@vincenz.de 


\section{Graphical Abstract}

\section{The impasse on overall survival in oncology reimbursement decision-making. How can we resolve this?}

1. The impasse

Wanting mature os data

Payers favor mature OS data, but it may not be available at the time they make decisions

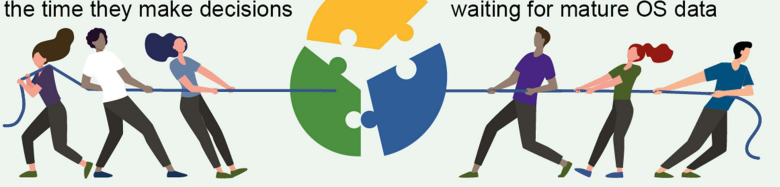

2. The scenarios

We note three scenarios with different levels of OS data maturity being available at drug launch

Particularly in early-stage disease, it may take years to prove survival benefit, or it may not be possible to attribute benefit to the new treatment because patients receive subsequent treatments

\section{The solutions}

Reimbursement systems need to evolve to align

with scientific advances because innovation is only meaningful if readily accessible to patients

Manufacturers and payers need to generate and appraise patient-relevant evidence from initial and follow-up analyses, with a focus on valuing pre-specified context-specific endpoints that stand in their own right

\section{The actions}

All stakeholders - patients, clinicians, manufacturers and payers - have a role to play:

- Agree on when mature OS data are to be expected for pricing and reimbursement appraisals

- Agree on the range of appropriate clinical endpoints that are informative in their own right

- Acknowledge that the highest level of statistical validation of surrogates is unlikely to be available in all circumstances

- Develop post-marketing real-world evidence or statistical modeling as required

- Consider using managed entry agreements when uncertainty is high and reappraise as more evidence becomes available

- Understand the value patients place on benefits from new therapies

\section{Plain Language Summary}

- Before patients can receive a new drug, regulators decide if it is safe and effective in treating the disease. Payers then decide if they will pay for the drug and, if so, at what price.

- For cancer drugs, payers prefer to make decisions based on overall survival (OS) results. This is a measure of how long patients live after starting treatment. However, it may take many years to collect OS data. For example, patients with early-stage cancers may live for a long time with effective treatment. In addition, benefit can be difficult to measure if patients subsequently receive numerous other therapies.

- If payers wait for long-term OS data, patients have to wait to access the new treatment, even when regulators have decided that it is safe and effective. As a result, some patients may die while waiting. On the other hand, if payers do not have long-term OS data, they risk paying for a treatment that is not as effective as they had hoped.

- We use our individual views as a patient advocate, clinician or health economist to suggest how payers, clinicians, manufacturers and patients can work together to help patients access potentially life-extending drugs and payers spend money wisely.

- We recommend using a range of clinical measures which can be measured earlier than OS, including delayed disease progression and improved quality of life.
- We propose using methods to manage uncertainty, for example agreements between payers and pharmaceutical companies that are based on patient outcomes.

- We suggest that payers evolve their decision-making so it aligns with advances in science. Innovation in new treatments is only meaningful if readily accessible to patients.

\section{Introduction}

Extending overall survival (OS) is an important treatment goal for many therapies in oncology. In clinical trials, OS is widely considered as the endpoint that is most valued by patients, clinicians, regulators and payers (see Box 1 for common definitions). However, mature data on OS (eg, the median) are often not available at the time when reimbursement authorization decisions for new therapies are being made. In early-stage disease, when survival times are longer, proving an OS benefit can take a long time or may not be possible at all as therapies become more effective. In other cases, when cancer is advanced and a cure is unlikely, many patients will not survive long and OS data may be available relatively rapidly. However, even in late-stage disease, it may be difficult to interpret these data because it is hard to separate out the effect of a new treatment on OS when multiple subsequent lines of therapy have been used, or when patients on standard of care cross over to the new treatment either 


\section{Box I Definitions}

Regulator: here, refers to medicines licensing agencies, such as the US Food and Drug Administration (FDA) and the European Medicines Agency (EMA).

Payer: here, refers to post-regulatory agencies involved in the funding and reimbursement of healthcare services or drugs, such as national health technology assessment (HTA) agencies and local funding/access decision-makers.

Endpoints: used in a clinical trial setting to evaluate whether a drug provides a clinical benefit. ${ }^{13}$ See the CBER/CDER guidelines for an overview for cancer drugs and biologics. ${ }^{14}$ Intermediate or biomarker endpoints can be endpoints in their own right or can be considered as surrogates when used to substitute for OS or other endpoints in clinical trials.

Overall survival (OS): the time from randomization until death from any cause. Maturity in OS is often defined by median OS, the time at which the Kaplan-Meier survival curve crosses the $50 \%$ cumulative survival probability. ${ }^{15}$ Note that median OS does not always fully assess the OS benefit, for example when a proportion of patients has a long survival time ('tail of the curve' phenomenon; eg, in immuno-oncology trials). ${ }^{16}$ In these cases, landmark analysis can be used. $^{17}$

Surrogacy: endpoints can substitute for OS or other final endpoints if they are validated, ie they have shown statistical correlation with treatment effect for the specific setting and patient population. ${ }^{18}$ This means that an endpoint may be a surrogate in one disease setting but not another.

Managed entry schemes: financial agreements between manufacturers and payers to allow introduction of innovative medicines while managing uncertainty around the treatment's financial impact or clinical evidence. Managed entry schemes may encompass patient access schemes, risk-sharing agreements, payment by results and performance-based risk-sharing agreements.

Abbreviations: CBER, Center for Biologics Evaluation and Research; CDER, Center for Drug Evaluation and Research.

during or after the trial. ${ }^{1}$ A tension then emerges between wanting to know whether, and by how much, a new treatment impacts on OS and needing to wait, sometimes for years, to obtain this information; or possibly never knowing with certainty. This tension is particularly acute for payers; given their primary objective of achieving the most health gain from their available budget, they need to be confident that the new treatment is providing value in terms of length of life, quality of life (QoL) or, ideally, both. There are strong opinions about approval and reimbursement in the absence of mature OS data, reflecting the high stakes at play, with concerns over delay in patient access set against concerns about the potential for treatments not to fully provide this anticipated benefit after longer follow-up and mature data. ${ }^{2,3}$ As payers have accountability to weigh the patient health benefit of new medicines against budget requirements, we see differences in expectations for OS among payers, and between payers and other stakeholders. ${ }^{4-6}$ In some cases, we see that regulators, clinicians and patients place different values on non-OS endpoints from payers. ${ }^{3,7,8}$ This can lead to delayed access to regulatory-approved novel treatments, and in some cases eligible patients could die while these decisions are being made. ${ }^{9}$ Time to reimbursement can differ significantly across health systems ${ }^{10}$ and some countries have implemented schemes to shorten the time to access; ${ }^{11}$ however, schemes cover only some geographic regions and selected patients and are often not directly related to how payers appraise endpoints beyond OS.

Addressing and resolving the "OS impasse" is now more important than ever. Science is moving ahead quickly: our understanding of the complex molecular biology underlying different cancers is growing, and innovative, often personalized, new treatments are being developed. These advances, combined with earlier diagnosis and treatment, mean that patients are living longer with cancer or following cancer and it is increasingly difficult to present mature OS data at regulatory approval. Payer approval still, however, remains a requirement for translating innovation into better outcomes for patients and healthcare systems. ${ }^{12}$

We have developed this position paper, taking our individual views gained from our experience as a patient advocate, clinician or health economist supported by evidence from the literature, and we focus on one aspect of this "OS impasse". We look at payer decision-making in the absence of mature OS data, considering when and how non-OS trial outcomes could inform decisions and how uncertainty can be addressed beyond the trial. Our aim is collaborative, to work alongside payers and other stakeholders to reach a consensus on when it is appropriate to consider alternative endpoints in place of OS if mature OS data from clinical trials are not reasonably available; this means ensuring that evidence is relevant and that the uncertainty and risks faced by patients, clinicians, payers and manufacturers is mitigated. Our focus is evidence and endpoints, and our aim is for these considerations to apply across appraisal systems, whether based on costeffectiveness or clinical/comparative effectiveness. While acknowledging the importance of drug pricing in decisionmaking and the trends in healthcare expenditure on cancer treatments, ${ }^{13}$ we do not address these aspects in this paper. 


\section{Decisions are Increasingly Being Made Without Mature OS Data}

Many regulatory and payer agencies consider OS as the most reliable and preferred endpoint (Table 1). ${ }^{14,19}$ Regulators acknowledge that the disease setting determines how endpoints can be considered for approval - the US Food and Drug Administration (FDA) notes that endpoint selection is highly dependent upon factors such as effect size and duration, depth of response, available therapy, location of disease, and the consequences of delaying or preventing disease

Table I Sample of Regulators', Payers' and Clinical Societies' Positions on Types of Endpoints

\begin{tabular}{|c|c|}
\hline Agency Region/Country & Stated Position \\
\hline \multicolumn{2}{|l|}{ Regulators } \\
\hline EMA, $2017^{19}$ Europe & $\begin{array}{l}\text { - OS is the most persuasive endpoint } \\
\text { - Prolonged PFS or DFS are in most cases considered relevant measures of patient benefit, but } \\
\text { the magnitude of the treatment effect should be sufficiently large to outbalance toxicity and } \\
\text { tolerability problems }\end{array}$ \\
\hline FDA, $2018^{14}$ USA & $\begin{array}{l}\text { - OS is the most reliable and preferred cancer endpoint } \\
\text { - Endpoints based on tumor assessments (DFS/EFS, ORR, CR, TTP and PFS) can be important }\end{array}$ \\
\hline \multicolumn{2}{|l|}{ Payers and HTA agencies } \\
\hline EUnetHTA, $2015^{29}$ Europe & $\begin{array}{l}\text { - OS is the preferred clinical endpoint } \\
\text { - PFS is an acceptable endpoint } \\
\text { - for cancer trials in the adjuvant, but not the metastatic, setting } \\
\text { - if there is a strong association between the effect of the surrogate and the effect on the } \\
\text { final endpoint of interest }\end{array}$ \\
\hline ICER, $2020^{30}$ USA & $\begin{array}{l}\text { - Direct OS measurement preferred } \\
\text { - If surrogate outcomes are used, they need to be validated }\end{array}$ \\
\hline IQWiG, $2017^{31}$ Germany & $\begin{array}{l}\text { - Most endpoints are not good surrogates for OS and may be unreliable and misleading } \\
\text { - Surrogates are only considered if they have been validated by appropriate statistical methods } \\
\text { within a sufficiently restricted patient population and within comparable approved interventions }\end{array}$ \\
\hline NICE, $2012,{ }^{32} 2019^{33, a}$ England & $\begin{array}{l}\text { - Surrogates may be used to infer the effect of treatment on mortality and HRQoL, if evidence in } \\
\text { support of surrogacy is provided } \\
\text { - A modeling framework is required to establish the strength of the surrogate relationship } \\
\text { between the treatment effects on the surrogate and the final outcome, and to explore the } \\
\text { uncertainty related to it }\end{array}$ \\
\hline PBAC, $2016^{34}$ Australia & - Submissions should not rely on surrogacy for effectiveness \\
\hline \multicolumn{2}{|l|}{ Clinical societies } \\
\hline $\begin{array}{l}\text { ASCO Value Framework Net Health Benefit } \\
\left(\text { ASCO-NHB) }{ }^{35} \text { USA }\right.\end{array}$ & $\begin{array}{l}\text { Assesses the relative value of cancer therapies by assigning a net health benefit score, depending } \\
\text { on the treatment's clinical benefit, toxicity and symptom palliation } \\
\text { - The framework puts weight on OS and toxicity; therapies without significant OS or DFS/EFS } \\
\text { benefit (improved median values or hazard ratios) are marked down } \\
\text { - Bonus points are awarded for tail of the OS curve }\end{array}$ \\
\hline $\begin{array}{l}\text { ESMO Magnitude of Clinical Benefit Scale } \\
(\text { ESMO-MCBS })^{36,37} \text { Europe }\end{array}$ & $\begin{array}{l}\text { Ranks cancer treatments by benefit, ie whether patients lived longer (improvement in OS or } \\
\text { surrogate for OS) or better (improvement of QoL or surrogate for QoL, or reduced toxicity) } \\
\text { - Treatments without mature OS data or those without OS as an endpoint are scored lower }\end{array}$ \\
\hline
\end{tabular}

Notes: aNew guidance is being developed and is, at the time of writing, in the consultation phase. ${ }^{38 .}$

Abbreviations: ASCO, American Society of Clinical Oncology; CR, complete response; DFS, disease-free survival; EFS, event-free survival; EMA, European Medicines Agency; ESMO, European Society for Medical Oncology; EUnetHTA, European Network for Health Technology Assessment; FDA, Food and Drug Administration (USA); HRQoL, health-related quality of life; HTA, health technology assessment; ICER, Institute for Clinical and Economic Review (USA); IQWiG, Institut für Qualität und Wirtschaftlichkeit im Gesundheitswesen (Germany); NICE, National Institute for Health and Care Excellence (England); ORR, overall/objective response rate; OS, overall survival; PBAC, Pharmaceutical Benefits Advisory Committee (Australia); PFS, progression-free survival; QoL, quality of life; TTP, time to progression. 
progression or delaying use of subsequent toxic therapies. ${ }^{14}$ These considerations are arguably broader than those employed by payers, and regulatory decisions are made without mature OS data for the majority of FDA and European Medicines Agency (EMA) oncology approvals. More than $70 \%$ of FDA adult cancer drug approvals between 2006 and 2017 were based on progression-free survival (PFS) and relapse-free survival. ${ }^{6}$ Similarly, an analysis of EMA approvals for oncology treatments between 2014 and 2017 showed that in 34 of 88 (39\%) marketing authorization applications, OS data were immature at the time of approval. ${ }^{20}$ Payer guidelines have stated a clear preference for mature OS data when appraising cancer drugs for reimbursement (Table 1). Across cancer types, payer organizations (eg, Germany's Federal Joint Committee [G-BA], the Spanish Agency of Medicines and Medical Products [AEMPS] and England's National Institute for Health and Care Excellence $[\mathrm{NICE}])^{21-26}$ have critiqued the absence of mature OS data when appraising new therapies, in some instances denying, restricting or delaying patient access; in other instances, providing access despite criticizing the evidence. ${ }^{27,28}$

\section{Consequences of Decision-Making in the Absence of Mature OS Data}

When improvement in OS is an important goal of therapy, decisions made in the absence of mature OS data come with risk and uncertainty, which can take different forms for different stakeholders. When the decision leads to no or delayed access, some patients can die waiting for OS to be demonstrated (see Box 2 for an example), while others can lose the option to benefit from potentially life-

\section{Box 2 Example for Impact of Earlier Time to Reimbursement for} Patients

An analysis published by the European Federation of Pharmaceutical Industries and Associations (EFPIA) in 2020 estimated the impact on patients of time to reimbursement in six European countries. ${ }^{9}$ Compared with the actual situation, it was estimated that very early reimbursement (at the time of European marketing authorization) of midostaurin for acute myeloid leukemia (AML) would have led to 1689 additional patients receiving treatment, living for an additional 6910 years; less rapid access (180 days after marketing authorization) was estimated to lead to 369 more patients receiving treatment, living for an additional 1509 years.

Note that an EU Transparency Directive stipulates that decisions on joint pricing and reimbursement of medicines should be made within 180 days of dossier submission. ${ }^{46}$ extending future treatment (loss of option value). ${ }^{39,40}$ This can lead to patients, clinicians and payers accepting methods to manage uncertainty. Both the American Society of Clinical Oncology (ASCO) and the European Society for Medical Oncology (ESMO) have developed tools to help assess the relative benefit of cancer therapies (see Table 1). However, these frameworks do not directly address how to enable patient access without mature OS data, in part because they are not regularly used by healthcare decision-makers, but also because of the focus on trial data rather than evidence beyond trials. Several countries have implemented schemes to shorten the time to access, such as reimbursement before the pricing decision (eg, in Germany) or early access (eg, Temporary Use Authorizations in France); elsewhere, coverage may be gained rapidly following regulatory approval (eg, in the USA and Japan). ${ }^{11}$ Some countries have schemes to address uncertainty specifically around cancer drugs (eg, Cancer Drugs Fund in the UK). However, we note that these schemes cover only some geographic regions and selected patients, and are not directly related to how payers appraise endpoints beyond OS.

Although there have been advances in supporting patients in accessing new cancer medicines, we must acknowledge that early access comes with the risk of patients experiencing adverse events or inconvenience for no meaningful gain in length or QoL. Some of these risks can be mitigated through rescinding approval for drugs that do not show sustained patient benefit. This was seen in early 2021 when approval was withdrawn across a limited number of indications granted through the FDA Accelerated Approval Program, for which the drugs had not met post-marketing requirements. ${ }^{41,42}$

There are also financial consequences to decisionmaking. A consequence of reimbursement when value is not established is the use of healthcare budget that could have been used on more effective therapies. $2,43,44$ A further financial consequence of delayed access is the lower than expected revenue for the manufacturer; this is felt immediately by the manufacturer but also has a longer term impact on patients because it puts at risk the development of new, innovative drugs, which is often costintensive. $^{45}$

To manage risks from payer coverage of new treatments while supporting early access, when possible, we first need to agree when it is reasonable to expect mature OS data and when it may not be. 


\section{Considering When Mature OS Data are, or are Not, Expected for Initial Reimbursement Decisions}

There can be no simple rule as to when payers should expect mature OS data. However, considerations based on criteria such as cancer stage/disease setting, treatment efficacy and trial design can help to inform when demonstrating an OS benefit may be difficult to achieve. We have suggested considerations that might be applicable, to differing extents, across many clinical settings (Table 2). We have combined these and outlined three broad scenarios regarding expectations of mature OS data being available.

- Scenario 1: Median OS data are available at first pricing and reimbursement negotiations. Examples of this scenario are metastatic cancers with few treatment options, or rapidly progressing disease such as small-cell lung cancer. In such scenarios, median OS data are regarded as sufficiently mature by many payers, and final survival may be estimated through statistical extrapolation of survival curves. Other approaches to inform decisions in the absence of mature OS data are often not required in this scenario. Payers may still consider the patient relevance of non-OS endpoints, such as PFS, disease-free survival (DFS), QoL and toxicities.

- Scenario 2: Limited OS data are available at first pricing and reimbursement negotiations, and mature OS data are expected within the therapy life cycle (before the therapy is off-patent or superseded). Examples based on today's treatments are metastatic diseases with several lines of therapy still available or progressive diseases, such as locally advanced non-small-cell lung cancer (NSCLC), after chemoradiation. In such scenarios, payers should consider the patient relevance of non-OS endpoints, such as PFS, DFS, QoL and QoL related to toxicities. Other approaches include statistical inference to estimate OS, surrogacy for OS, utilizing RWE and managed entry agreements, with the choice of approach partly depending on the maturity of the data. Treatment crossover and switching can make statistical inference and other approaches more challenging.

- Scenario 3: Incomplete or no OS data are available at first pricing and reimbursement decisions, or during the therapy life cycle. Examples include indolent and potentially curative cancers such as early luminal A breast cancer, and trials in which early unblinding prevents OS data maturity from being reached. In such scenarios, the intrinsic value of context-relevant and patient-relevant endpoints that reflect how the patient feels or functions ${ }^{47}$ (eg, PFS, DFS and QoL or patientreported outcomes) will need to gain increasing weight in payer assessments. This can be as endpoints in their own right, as reflected in the ASCO Value Framework Net Health Benefit (ASCO-NHB) ${ }^{35}$ and ESMO Magnitude of Clinical Benefit Scale (ESMOMCBS), ${ }^{36,37}$ with assessment of which endpoints are most appropriate in a given disease context (preferably through a well-designed core outcome set). ${ }^{48}$ Surrogates for OS may also be appropriate; however, approaches to assess the correlation between surrogate endpoints and OS may rely on data from similar drugs or drug classes or have high associated uncertainty.

Across these scenarios, we note the roles the product label and European Public Assessment Report (EPAR) can play in providing clear, non-technical communication of the available OS data, describing the magnitude of benefit as well as the level of immaturity or confounding. We also note the role of the manufacturer in continued OS data collection and communication after regulatory and payer approval, within or beyond the trial when feasible. We endorse the use of robust trial designs to limit bias and to achieve a high methodological quality of pivotal trials, which has been widely debated in the literature. ${ }^{49,50}$ This includes ensuring OS data are available for safety assessment in relevant settings.

We propose that when OS data are not available or are limited at the time of the initial pricing and reimbursement decision, this should not itself prevent patient access, provided other meaningful and patient-centered benefits have been demonstrated, which have been pre-specified and included in the trial design. We need to agree on how to appraise drugs in these scenarios, noting that the need for decision-making without mature OS data is likely to increase in the future; particularly as therapies are used in earlier disease settings or become curative. This means a specific disease setting could evolve as the science advances, from one in which mature OS data are expected at launch to one in which collection of mature OS data are not considered feasible. This development could resemble changes seen in other disease areas, for example human immunodeficiency virus (HIV)/acquired immune deficiency syndrome (AIDS) (Box 3). 
Table 2 Considerations When Proving an OS Benefit is Difficult to Achieve or is Not of Primary Relevance to Patients

\begin{tabular}{|c|c|}
\hline Consideration & Factors and Examples \\
\hline Indolent disease therapies & $\begin{array}{l}\text { Patients will have long life expectancy, with OS highly relevant to patients but requiring a long duration } \\
\text { of follow-up to assess } \\
\text { For example, in chronic lymphocytic leukemia, life expectancy often reaches } 10 \text { years or more; here, PFS } \\
\text { and minimal residual disease are relevant endpoints }{ }^{51,52} \text { accepted by regulators }\end{array}$ \\
\hline Early-line therapies & $\begin{array}{l}\text { Patients have long life expectancy requiring a long wait to assess OS data, which may be confounded by } \\
\text { treatment with other therapies } \\
\text { Patients may value highly time before progression, for example, if they experience more symptoms or } \\
\text { receive more intensive therapies post-progression (eg, chemotherapy after endocrine-based therapy in } \\
\text { HR+ metastatic breast cancer), making endpoints such as PFS/DFS highly relevant }\end{array}$ \\
\hline $\begin{array}{l}\text { Palliative therapies or when OS is not } \\
\text { the goal }\end{array}$ & $\begin{array}{l}\text { Patients may prioritize experiencing fewer side effects or a better QoL and symptom improvement over } \\
\text { OS gain. So, although OS gain may be shown, it is important that trials evaluate endpoints relevant for } \\
\text { the specific setting and therapy } \\
\text { For example, in pancreatic cancer, relief of disease-related symptoms was measured using clinical benefit } \\
\text { response, in which gemcitabine achieved } 24 \% \text { compared with } 5 \% \text { for } 5 \text {-FU }(p=0.0022) \text {. The median } \\
\text { survival was } 5.65 \text { months for gemcitabine compared with } 4.41 \text { months for } 5 \text {-FU }(p=0.0025)^{53}\end{array}$ \\
\hline Early unblinding & $\begin{array}{l}\text { Trials that show an early signal of strong benefit may be stopped early and automatically unblinded, }{ }^{54} \\
\text { limiting the ability to show OS gain, because the control group will be treated differently after unblinding } \\
\text { (eg, crossover will be allowed) } \\
\text { For example, in the Phase } 3 \text { trial of osimertinib, unblinding was recommended by an Independent Data } \\
\text { Monitoring Committee after the drug showed "overwhelming efficacy" in patients with Stage IB, II and } \\
\text { IIIA epidermal growth factor receptor-mutated NSCLC with complete tumor resection }{ }^{55}\end{array}$ \\
\hline Confounded treatment effect & $\begin{array}{l}\text { Trials with crossover, or in which the study treatment is followed by several lines of different therapies, } \\
\text { can have a confounding treatment effect on OS, making it difficult to identify how much OS gain can be } \\
\text { attributed to the treatment of interest; although statistical methods have been developed }{ }^{56,57} \\
\text { For example, in a phase } 3 \text { trial with the PARP inhibitor olaparib in patients with ovarian cancer with } \\
\text { a BRCAI or BRCA2 mutation, median OS did not reach significance vs placebo (HR for death, } 0.74 ; 95 \% \\
\text { Cl: } 0.54-1.00 ; p=0.054) \text {; after adjusting for the } 38 \% \text { of patients in the placebo group who received } \\
\text { subsequent PARP inhibitor therapy, the HR was } 0.56(95 \% \mathrm{Cl}: 0.35-0.97)^{58}\end{array}$ \\
\hline $\begin{array}{l}\text { Rare cancers (single-arm trials or trials } \\
\text { with low power) }\end{array}$ & $\begin{array}{l}\text { Difficulty in recruiting patients to trials can lead to single-arm trials or trials with low power that are } \\
\text { unlikely to show statistical differences in OS between treatments } \\
\text { As well as rare cancers, use of very detailed inclusion and exclusion criteria (eg, requirements for the } \\
\text { presence of baseline predictive factors or previous therapies) or selection for rare mutations can lead to } \\
\text { small population sizes and low power } \\
\text { For example, the FDA granted accelerated approval for brentuximab vedotin in anaplastic large cell } \\
\text { lymphoma based on a single-arm multicenter clinical trial of } 58 \text { patients after failure of at least one prior } \\
\text { chemotherapy regimen. The primary endpoint, objective response rate by independent review, was } 86 \% \\
(95 \% \mathrm{Cl}: 77-95 \%) \text { with a median duration of } 12.6 \text { months }\end{array}$ \\
\hline Curative or approaching cure & $\begin{array}{l}\text { For curative therapies, the active treatment arm of the clinical study would reflect the normal life } \\
\text { expectancy of the population } \\
\text { For example, the life expectancy of men with a diagnosis of testicular cancer at } 30 \text { years of age is } 2 \text { years } \\
\text { less than cancer-free men of the same age, and the difference approaches zero with increasing survival } \\
\text { time }{ }^{60} \\
\text { As the science of oncology advances, we would expect to see more therapies and disease areas fall into } \\
\text { this scenario }\end{array}$ \\
\hline
\end{tabular}

Abbreviations: 5-FU, 5-fluorouracil; Cl, confidence interval; DFS, disease-free survival; FDA, Food and Drug Administration (USA); HER2, human epidermal growth factor receptor 2; HR, hazard ratio; HR+, hormone receptor-positive; NSCLC, non-small-cell lung cancer; OS, overall survival; PARP, poly (ADP-ribose) polymerase; PFS, progression-free survival; QoL, quality of life. 
Box 3 Example of a Progressive Shift Away from OS

When AIDS was a life-threatening diagnosis with extremely poor survival, survival was the most relevant endpoint. ${ }^{61}$ Through advances in antiretroviral therapy, HIV infection is now a chronic, manageable condition, and life expectancy is similar to the general population. ${ }^{62}$ Today, CD4 cell count and viral load are considered clinically relevant endpoints, and QoL outcomes are prioritized. ${ }^{63,64}$ It is likely that many cancers will also become chronic diseases with long survival with novel therapies.

Abbreviations: AIDS, acquired immune deficiency syndrome; HIV, human immunodeficiency virus; OS, overall survival; QoL, quality of life.

\section{Supporting Decision-Making in the Absence of Mature OS Data}

When no or immature OS data are expected at the time of the initial reimbursement decision, alternative approaches for assessing clinical benefit are required and appropriate endpoints should be pre-specified during clinical trial design. We suggest approaches below, noting their applicability according to OS data availability. We do not expect all approaches will be used across the three scenarios described previously - for each scenario there will be a limit to how much value additional information will provide, relative to the financial impact of the decision (we note, value of information analysis can in some circumstance help to assess the expected gain from reducing uncertainty and the cost-effectiveness of further research). ${ }^{65}$

\section{Context-Relevant Endpoints}

Endpoints that measure disease status and/or how patients feel and function have intrinsic value and stand "in their own right", rather than through an association of how long the patient survives (ie, OS). For example, patients may value relief from pain, avoidance of particular symptoms or adverse events, or simply being "cancer free", and these preferences should be ascertained for distinct patient groups. Patient-relevant endpoints can be based on tumor assessments, symptom or toxicity assessment, or QoL, with these endpoints being powered in clinical trials and/ or being part of a core outcome set (Table 3). ${ }^{48} \mathrm{We}$ recommend defining the importance of context-relevant endpoints by setting (as outlined in Table 2) through

Table 3 List of Endpoints to Consider as Potential Valid Measures of Efficacy

\begin{tabular}{|c|c|}
\hline Endpoint & Definition \\
\hline (p)CR & (Pathologic) complete response: defined as the disappearance of all signs of cancer in response to treatment \\
\hline $\begin{array}{l}\text { EFS/DFS/ } \\
\text { RFS }\end{array}$ & $\begin{array}{l}\text { Event-free survival/disease-free survival/relapse-free survival: defined as the time from cancer treatment end to the appearance of } \\
\text { first signs or symptoms of that cancer, or the appearance of first complications that the treatment was intended to prevent/delay, or } \\
\text { death from any cause } \\
\text { EFS is normally used in the neoadjuvant setting and DFS is used in the adjuvant setting }\end{array}$ \\
\hline iDFS/MFS & $\begin{array}{l}\text { Invasive disease-free survival: defined as the time between randomization and first invasive disease recurrence; primarily used in } \\
\text { studies of patients with breast cancer } \\
\text { Metastasis-free survival: defined as the time from start of treatment for cancer that a patient is still alive and the cancer has not } \\
\text { spread to other parts of the body; primarily for patients with prostate cancer }\end{array}$ \\
\hline MRD & $\begin{array}{l}\text { Minimal residual disease: defined as the small proportion of cancerous cells that may remain after treatment when the patient shows } \\
\text { no signs or symptoms of the disease; primarily used in trials with patients with hematologic malignancies }\end{array}$ \\
\hline ORR & $\begin{array}{l}\text { Overall/objective response rate: defined as the proportion of patients with a confirmed response of complete or partial response } \\
\text { (usually tumor size reduction) }\end{array}$ \\
\hline PRO & $\begin{array}{l}\text { Patient-reported outcome: can directly measure clinical benefit (severity of cancer symptoms) } \\
\text { Evaluated using validated measures that are routinely incorporated into clinical trial design, such as EQ-5D or SF-36 } \\
\text { Some endpoints include a QoL component, such as TWiST (time without symptoms of disease and toxicity of treatment) or QAPFS } \\
\text { (quality-adjusted PFS) } \\
\text { NB: capturing QoL endpoints and powering for them may not always be feasible in RCTs; a high follow-up rate is needed for } \\
\text { questionnaires }\end{array}$ \\
\hline PFS & $\begin{array}{l}\text { Progression-free survival: defined as the time from randomization to the date of first disease progression or death } \\
\text { Related measures include PFS2 (time from randomization to tumor progression on next-line treatment or death from any cause), } \\
\text { TTD (time to treatment discontinuation) and TTNT (time to next treatment) }\end{array}$ \\
\hline
\end{tabular}

Notes: Based on the FDA's table of important cancer approval endpoints ${ }^{79}$ and the National Cancer Institute dictionary of cancer terms. ${ }^{80 .}$

Abbreviations: EQ-5D, 5-dimension EuroQol questionnaire; FDA, Food and Drug Administration (USA); PRO, patient-reported outcome; QoL, quality of life; RCT; randomized controlled trial; SF-36, 36-item short-form health survey. 
consultation with patients. We note also that statistical approaches such as generalized pairwise comparison can be used to assess net benefit across several outcomes allowing secondary endpoints a greater weight in the decision. ${ }^{66}$ Furthermore, we encourage HTA agencies to define and adopt prespecified core sets of outcomes that cover a variety of target domains beyond mature OS. Asking patients what they value and quantifying the outcome provides meaningful information for decisionmaking and we discuss tumor assessment, QoL, symptoms and toxicity endpoints below, noting that other endpoints can be considered context relevant.

Tumor assessment: endpoints related to disease can have a strong biological rationale - for example, showing that a tumor has responded to treatment or not progressed, and this rationale can be very specific to the setting. A demonstrable response to therapy can be associated with improved symptoms and functioning and bring other advantages, such as significant influence on quality of life in metastatic breast cancer, ${ }^{67}$ or strong psychological benefit in neoadjuvant breast cancer (from achieving pathologic complete response, as reported during an HTA decision committee). ${ }^{68,69}$ Conversely, tumor progression was associated with substantial worsening in QoL in advanced breast, pancreatic, lung or colorectal cancer. ${ }^{70}$

Similarly, event-free survival and DFS can be direct measures of clinical benefit (if toxicity does not cause detriment in QoL), for example, indicating longer time free of metastasis. ${ }^{71}$

QoL, toxicity and symptoms: living better is an important potential benefit from cancer treatment, alongside the potential to live longer, ${ }^{37}$ and QoL is a recognized patient-relevant endpoint in its own right. ${ }^{31}$ Endpoints relating to toxicity and symptom control can also be highly relevant when measuring features of disease or treatment that are important to patients. ${ }^{69}$ The analysis and interpretation of QoL measures can, however, limit comparison between trials. ${ }^{72}$ For use in decision-making, QoL endpoints need to be prospectively incorporated into the trial to answer a well-defined research question, have adequate statistical power and limited missing data points, and the instrument selected according to the patient population and the objectives of treatment (eg, see considerations in ovarian cancer). ${ }^{72-75}$ We note that patient QoL and function can also have a profound impact on caregivers, with deteriorating functionality associated with increased caregiver burden. ${ }^{76}$ When considering the toxicity of a treatment, the severity, timing and duration of adverse events can all be important, and we need to work with patients to understand these factors in different disease and treatment settings. ${ }^{77}$ In some settings, patients are willing to trade significant PFS time for reductions in treatment-related toxicity, showing the importance of toxicity endpoints. $^{78}$

Overall, we reinforce the importance of reaching international agreement on the use of accepted patient-relevant endpoints so that prospective trials can be planned, based on criteria accepted across HTAs. The effect size for decision-making will depend on the setting and is not discussed here.

\section{Surrogacy}

Endpoints can substitute for OS or other endpoints if they are validated, ie, they have shown statistical correlation with treatment effect for the specific setting and patient population. In some settings, the validity of an endpoint as surrogate for OS has been shown; ${ }^{81}$ however, surrogacy in one disease setting will rarely translate to other settings. Surrogacy is generally accepted by HTA bodies when validated for the treatment, setting and patient group of interest. $^{82}$ However, in practice, surrogacy for OS is often difficult to prove at the time of initial reimbursement - it is seldom possible to show a robust correlation between the treatment effect on the surrogate and the treatment effect on OS within the same treatment class and indication especially if the new treatment is the first in class. We recommend further research into surrogate validation, focusing on sharing trial participant data and on reducing prediction uncertainty. ${ }^{18,83}$ Understanding which endpoints have been used, when standardized definitions are not followed, is an additional complexity. We recommend the use of standard definitions for endpoints to optimize the comparison of studies and improve surrogate analyses.

There is general agreement on validation methodology using a three-step process of establishing the level of evidence, assessing the strength of association, and quantifying the relation between the surrogate and the final outcome. $^{84}$ Full statistical validation is required before a surrogate for OS can be recognized, although HTA organizations' handling of surrogate endpoints varies greatly, with inconsistency in what is considered a robust validation approach. ${ }^{85-87}$ Indeed, most HTA agencies do not provide guidance on how to evaluate surrogates for OS or other non-OS outcomes. ${ }^{82}$ We suggest that the framework for validation requires some flexibility in settings in which clinical evidence is limited to a small number of 
studies, or a single study, such as can be the case for therapies that are first-in-class, for rare tumors or for specific genotypes, in which the highest level of statistical validation may not be possible.

\section{Statistical Inference}

When limited OS data are available, statistical methods can be used to adjust for uncertainty in the available evidence. Methods to adjust for treatment crossover can provide close approximations of the true treatment effect, limiting uncertainty from confounding; ${ }^{56,57}$ however, these techniques are by no means perfect, and will not always remove confounding variables, so they are to be used with caution. Extrapolation of the Kaplan-Meier curve for OS can estimate treatment effect beyond the time frame of the trial and is a method accepted by some HTA agencies. ${ }^{1}$ There is evidence that this method can be accurate and even conservative in certain settings, with the OS benefit projections being less than the true benefit; ${ }^{88}$ a study summarizing 11 cancer immunotherapy submissions to NICE found that the initial extrapolation tended to underestimate OS by $0.4-2.7 \%$, depending on the assessment method used. ${ }^{89}$ There was a similar finding when replicating an economic model that was based on extrapolated OS data (for relapsed, platinum-sensitive, BRCA mutation-positive ovarian, fallopian tube and peritoneal cancer) in which the patient benefit estimated with early data $(\sim 3.5$ years of follow-up) was approximately half the patient benefit estimated when more mature data were available ( $\sim 6.5$ years of follow-up). ${ }^{90}$

\section{Use of Additional Evidence Gathered Outside of Clinical Trials}

RWE and post-marketing data collection are becoming increasingly important when limited OS data are available from a trial (see Box 4 for an example). These are expected after the initial payer decision, and at times are coupled with

\section{Box 4 Example of Clinical Benefit Shown with RWE}

While the PALOMA-2 study of first-line palbociclib plus letrozole in patients with HR+/HER2 - metastatic breast cancer is ongoing and yet to report mature OS data, a significant OS benefit was shown from an analysis of electronic health records from a heterogeneous, realworld population (HR vs letrozole, 0.58 [95\% Cl: 0.46-0.73]) and among subgroups. ${ }^{97}$

Abbreviations: $\mathrm{Cl}$, confidence interval; HER2, human epidermal growth factor receptor 2; $\mathrm{HR}$, hazard ratio; $\mathrm{HR}+$, hormone receptor-positive; OS, overall survival; RWE, real-world evidence. a managed entry agreement. ${ }^{91,92}$ Data collected beyond the clinical trial setting, such as through central registries, are nonrandomized and can be subject to limitations, but can include more patients in the usual care setting who are potentially followed for longer than in a trial, reducing uncertainty from trial data ${ }^{93}$ and supporting treatment optimization..$^{94}$ Indeed, even when trial OS data are mature or not confounded, RWE remains informative for these reasons, identifying potential gaps between efficacy and effectiveness; for example, due to differences in patient characteristics and the delivery of care between settings. ${ }^{95}$ An ongoing challenge is to limit delays in starting real-world studies, with the aim to start collecting RWE close to the time of regulatory approval.

RWE can also support statistical inference to build predictive models of OS according to different patient profiles, and HTA agencies are actively encouraging early dialog to align on RWE data collection. ${ }^{96}$

\section{Managed Entry Agreement Schemes}

Managed entry agreements are an option to consider to grant patient access to promising therapies when uncertainty about patient benefit is high and the scheme is feasible. An example of this approach is provided by the post-2016 UK Cancer Drugs Fund, a financial program that allows initial access to a new, promising treatment while additional confirmatory evidence is being generated, often as RWE, with appraisal of this evidence a requirement for continued funding. This access is timelimited, and renewal is contingent on the manufacturer showing evidence of cost-effectiveness based on additional data. In France, the Temporary Authorization for Use (ATU) program allows access to new treatments before marketing authorization if the new therapy is for a serious or rare indication for which there are no other appropriate therapies available for in France.

\section{Next Steps: Evolving the HTA Process to Align with Advances in Oncology and to Focus on Patient Need}

We have combined the patient advocate, clinician and health economist viewpoints to suggest when and how trial outcomes other than OS could inform decision-making and how uncertainty can be addressed for investigational agents that have regulatory approval without mature OS data at the time of appraisal for reimbursement. Alignment among stakeholders is central to this and, as a first step, we suggest the 
following actions, with the starting point being that an absence of mature OS data is not a reason in itself to refuse patient access, providing there is robust evidence to support significant benefit to patients.

- Gain agreement between payers and manufacturers on when mature OS data are to be expected for pricing and reimbursement appraisals.

- Reach agreement among payers on the range of appropriate clinical endpoints (as outlined in Table 3) that are informative in their own right and find opportunities to engage patients in assessing the importance of these endpoints for example, in the context of core outcome sets, which should be prespecified.

- Acknowledge that the highest level of statistical validation of surrogates is unlikely to be available in all circumstances and initiate further research into trial data sharing and surrogate endpoint validation.

- Manufacturers to invest in developing evidence from post-marketing RWE or statistical inference to support and extend randomized controlled trial evidence, and payers to consider this evidence.

- Manufacturers and payers to use managed entry agreements when uncertainty is high and the agreement is feasible and to reappraise as more evidence becomes available.

- Consult with patients to understand the value people place on benefits from new therapies, measured using a range of endpoints.

Developing operational guidance tailored by disease and treatment setting (eg, for early- versus late-stage disease) could form an additional step, requiring active collaboration between payers, manufacturers, patients and clinicians. Guidance regarding decision-making without mature OS data might also require adaptation to the specific reimbursement processes (eg, by country). This guidance could help everyone involvedpayers, patients, clinicians and manufacturers - in making and understanding coverage and reimbursement decisions.

\section{Conclusions}

Our reimbursement systems should evolve to align with scientific advances in oncology. As treatments become increasingly effective and some move to being curative, we need new ways of assessing therapies without mature OS data to avoid lives being shortened unnecessarily or quality of life declining, through a lack of timely reimbursement. Fair reimbursement is important, valuing patient benefit as shown through prespecified endpoints, but reappraising as required by payers in case of ongoing uncertainty or failure to show a sustained benefit. We encourage manufacturers and payers to define flexible strategies for generating and appraising patient-relevant evidence and managing uncertainty when mature OS data are not to be expected at the time of reimbursement decision-making, and for both parties to routinely follow these strategies, as innovation is only meaningful if readily accessible to patients. We believe that the changes suggested above will promote thorough assessment and timely access to effective medicines.

\section{Acknowledgments}

The authors would like to thank Bengt Liljas, Alison Horsfield, Orkideh Malkoc and Jason Ward of AstraZeneca for their critical review of the manuscript, and Polly Field and Johanna Scheinost of Oxford PharmaGenesis for medical writing support.

\section{Author Contributions}

All authors made a significant contribution to the work reported, whether that is in the conception, study design, execution, acquisition of data, analysis and interpretation, or in all these areas; took part in drafting, revising or critically reviewing the article; gave final approval of the version to be published; have agreed on the journal to which the article has been submitted; and agree to be accountable for all aspects of the work.

\section{Funding}

This article was developed following a targeted literature review on the subject in 2019 and a series of virtual meetings with external experts held in June and July 2020, organized and sponsored by AstraZeneca. Medical writing assistance was provided by Oxford PharmaGenesis, funded by AstraZeneca. The authors have not received any payment from AstraZeneca for this work.

\section{Disclosure}

The views expressed in this article are the personal views of the authors and are not made on behalf of, or reflect the position of, the companies or organizations with which the authors are affiliated. M.P.L. participated in advisory boards for AstraZeneca, Daiichi Sankyo, Gilead, Eisai, Exact Sciences, Grünenthal, Lilly, MSD, Novartis, 
PharmaMar, Pfizer, Pierre Fabre and Roche; performed lectures for AstraZeneca, Eisai, Exact Sciences, Lilly, MSD, Novartis, Pfizer, Gilead, Pierre Fabre, Grünenthal and Roche; and received travel expenses from Pfizer and Roche. He is an editorial board member of medactuell (medac). O.C. did not receive any funding from AstraZeneca and participated in advisory boards for BresMed. She is currently funded by the European Union's Horizon 2020 research and innovation program under grant 779306 (COMED - Pushing the Boundaries of Cost and Outcome Analysis of Medical Technologies). W. C.N.D. is an employee of AstraZeneca. A.F. reports no competing interests. M.F. participated in and was reimbursed for advisory boards for AstraZeneca, GSK, Lilly, MSD, Novartis and Takeda; lectures for AstraZeneca; received speakers fee from GSK and ACT Genomics; received travel expenses from AstraZeneca; participated in a steering committee for AbbVie and a data monitoring committee for AGITG; and received research funding (institutional) from AstraZeneca, Beigene and Novartis. The authors report no other conflicts of interest in this work.

\section{References}

1. NICE Decision Support Unit. NICE DSU technical support document 16: adjusting survival time estimates in the presence of treatment switching; 2014. Available from: http://nicedsu.org.uk/wp-content/uploads/2016/03/ TSD16_Treatment_Switching.pdf. Accessed March 13, 2020.

2. Prasad V. Do cancer drugs improve survival or quality of life? BMJ. 2017;359:j4528. doi:10.1136/bmj.j4528

3. Sola-Morales O, Volmer T, Mantovani L. Perspectives to mitigate payer uncertainty in health technology assessment of novel oncology drugs. $J$ Mark Access Health Policy. 2019;7(1):1562861. doi:10.1080/ 20016689.2018.1562861

4. Pinto A, Naci H, Neez E, Mossialos E. Association between the use of surrogate measures in pivotal trials and health technology assessment decisions: a retrospective analysis of NICE and CADTH reviews of cancer drugs. Value Health. 2020;23(3):319-327. doi:10.1016/j. jval.2019.10.010

5. Ruof J, Knoerzer D, Dunne AA, Dintsios CM, Staab T, Schwartz FW. Analysis of endpoints used in marketing authorisations versus value assessments of oncology medicines in Germany. Health Policy (New York). 2014;118(2):242-254. doi:10.1016/j.healthpol.2014.08.004

6. Chen EY-S, Joshi SK, Prasad V. FDA acceptance of surrogate endpoints in later lines of therapy. J Clin Oncol. 2018;36(15_suppl):6517. doi:10.1200/JCO.2018.36.15_suppl.6517

7. Dilla T, Lizan L, Paz S, et al. Do new cancer drugs offer good value for money? The perspectives of oncologists, health care policy makers, patients, and the general population. Patient Prefer Adherence. 2015;10:1-7.

8. Looking to the future: oncology endpoints (summary report of a joint workshop held on of 3 July 2017 by the Academy of Medical Sciences and the Association of the British Pharmaceutical Industry); 2017. Available from: https://acmedsci.ac.uk/file-download/41135280. Accessed February 8, 2021.
9. Vintura. Every day counts: improving time to patient access to innovative oncology therapies in Europe; 2020. Available from: https://www.efpia.eu/media/578013/every-day-counts.pdf. Accessed January $12,2021$.

10. EFPIA. The root cause of unavailability and delay to innovative medicines: reducing the time before patients have access to innovative medicines; 2020. Available from: https://www.efpia.eu/media/ 554527/root-causes-unvailability-delay-cra-final-300620.pdf. Accessed April 20, 2021.

11. OECD. Addressing challenges in access to oncology medicines analytical report; 2020. Available from: https://www.oecd.org/ health/health-systems/Addressing-Challenges-in-Access-to-Oncology -Medicines-Analytical-Report.pdf. Accessed April 21, 2021.

12. Ciani O, Armeni P, Boscolo PR, Cavazza M, Jommi C, Tarricone R. De innovatione: the concept of innovation for medical technologies and its implications for healthcare policy-making. Health Policy Technol. 2016;5(1):47-64. doi:10.1016/j.hlpt.2015.10.005

13. Hofmarcher T, Lindgren P, Wilking N, Jonsson B. The cost of cancer in Europe 2018. Eur $J$ Cancer. 2020;129:41-49. doi:10.1016/j. ejca.2020.01.011

14. Food and Drug Administration. Clinical trial endpoints for the approval of cancer drugs and biologics - guidance for industry; 2018. Available from: https://www.fda.gov/media/71195/download. Accessed January 23, 2020.

15. Dudley WN, Wickham R, Coombs N. An introduction to survival statistics: Kaplan-Meier analysis. J Adv Pract Oncol. 2016;7(1):91-100.

16. Kaufman HL, Atkins MB, Subedi P, et al. The promise of immuno-oncology: implications for defining the value of cancer treatment. J Immuno Ther Cancer. 2019;7(1):129. doi:10.1186/ s40425-019-0594-0

17. Dafni U. Landmark analysis at the 25-year landmark point. Circ Cardiovasc Qual Outcomes. 2011;4(3):363-371.

18. Ciani O, Davis S, Tappenden P, et al. Validation of surrogate endpoints in advanced solid tumors: systematic review of statistical methods, results, and implications for policy makers. Int J Technol Assess Health Care. 2014;30(3):312-324. doi:10.1017/S0266462314000300

19. European Medicines Agency. Guideline on the evaluation of anticancer medicinal products in man; 2017. Available from: https://www. ema.europa.eu/en/documents/scientific-guideline/guidelineevaluation-anticancer-medicinal-products-man-revision-5_en.pdf. Accessed March 13, 2020.

20. Kordecka A, Walkiewicz-Zarek E, Lapa J, Sadowska E, Kordecki M. Selection of endpoints in clinical trials: trends in European marketing authorization practice in oncological indications. Value Health. 2019;22(8):884-890. doi:10.1016/j.jval.2019.03.007

21. Enzalutamide for hormone-relapsed non-metastatic prostate cancer. NICE technology appraisal guidance [TA580]; 2019. Available from: https://www.nice.org.uk/guidance/ta580. Accessed February 1, 2021.

22. Vismodegib for treating basal cell carcinoma. NICE technology appraisal guidance [TA489]; 2017. Available from: https://www. nice.org.uk/guidance/ta489. Accessed February 1, 2021.

23. Justification to the resolution of the Federal Joint Committee (G-BA) on an amendment of the pharmaceuticals directive (AM-RL): Annex XII - resolutions on the benefit assessment of medicinal products with new active ingredients according to section 35a SGB V palbociclib; 2017. Available from: https://www.g-ba.de/downloads/ 40-1465-4388/2017-05-18_AM-RL-XII_Palbociclib_D-264_TrG_ EN.pdf. Accessed February 1, 2021.

24. Justification to the resolution of the Federal Joint Committee (G-BA) on an amendment of the pharmaceuticals directive (AM-RL): Annex XII - resolutions on the benefit assessment of medicinal products with new active ingredients according to section 35a SGB $\mathrm{V}$ abemaciclib (breast cancer; in combination with fulvestrant); 2019. Available from: https://www.g-ba.de/downloads/40-14655714/2019-05-02_AM-RL-XII_Abemaciclib_D-401_TrG_EN.pdf. Accessed February 1, 2021. 
25. Informe de Posicionamiento Terapéutico de ixazomib (Ninlaro ${ }^{\circledR}$ ) en mieloma múltiple. [Therapeutic Positioning Report of ixazomib (Ninlaro ${ }^{\circledR}$ ) in multiple myeloma]; 2018. Available from: https:// www.aemps.gob.es/medicamentosUsoHumano/informesPublicos/ docs/IPT-ixazomib-Ninlaro-mieloma-multiple.pdf?x54046. Accessed February 1, 2021.

26. Informe de Posicionamiento Terapéutico de pembrolizumab $\left(\right.$ Keytruda $^{\circledR}$ ) en el tratamiento de Linfoma de Hodgkin. [Report on Therapeutic Positioning of pembrolizumab (Keytruda ${ }^{\circledR}$ ) in the treatment of Hodgkin's Lymphoma]; 2018. Available from: Available from: https://www.aemps.gob.es/medicamentosUsoHumano/ informesPublicos/docs/IPT-pembrolizumab-Keytruda-linfomaHodgkin.pdf?x98091. Accessed February 1, 2021.

27. Nutzenbewertungsverfahren zum Wirkstoff Bosutinib. [Benefit assessment procedure for the active ingredient bosutinib]; 2013 Available from: https://www.g-ba.de/bewertungsverfahren/nutzenbe wertung/68/. Accessed January 12, 2021.

28. Re-submission bosutinib; 2015. Available from: https://www.scottish medicines.org.uk/media/1353/bosutinib_bosulif_resubmission_ final_jan_2015_for_website.pdf. Accessed January 12, 2021.

29. EUnetHTA. Guideline: endpoints used for relative effectiveness assessment: clinical endpoints; 2015. Available from: https:// eunethta.eu/wp-content/uploads/2018/02/WP7-SG3-GLclin_endpoints_amend2015.pdf. Accessed January 23, 2020.

30. Institute for Clinical and Economic Review. ICER evidence rating matrix: a user's guide; 2020. Available from: https://icer.org/evi dence-rating-matrix/. Accessed January 6, 2020.

31. Institut für Qualität und Wirtschaftlichkeit im Gesundheitswesen. General methods (version 5.0); 2017. Available from: https://www.iqwig.de/down load/General-Methods_Version-5-0.pdf. Accessed January 23, 2020.

32. NICE Decision Support Unit. A review of studies examining the relationship between progression-free survival and overall survival in advanced or metastatic cancer; 2012. Available from: http://nicedsu. org.uk/methods-development/pfs-os/. Accessed January 23, 2020.

33. NICE Decision Support Unit. NICE DSU technical support document 20: multivariate meta-analysis of summary data for combining treatment effects on correlated outcomes and evaluating surrogate endpoints; 2019. Available from: http://nicedsu.org.uk/wp-content/uploads/2020/ 10/TSD-20-mvmeta-final.pdf. Accessed January 11, 2021.

34. Pharmaceutical Benefits Advisory Committee Appendix 5. Translating comparative treatment effects of proposed surrogate measures to target clinical outcomes; 2016. Available from: https://pbac. pbs.gov.au/appendixes/appendix-5.html. Accessed January 20, 2020.

35. Schnipper LE, Davidson NE, Wollins DS, et al. Updating the American Society of Clinical Oncology value framework: revisions and reflections in response to comments received. J Clin Oncol. 2016;34(24):2925-2934. doi:10.1200/JCO.2016.68.2518

36. Cherny NI, Dafni U, Bogaerts J, et al. ESMO-magnitude of clinical benefit scale version 1.1. Ann Oncol. 2017;28(10):2340-2366. doi:10.1093/annonc/mdx310

37. Cherny NI, Sullivan R, Dafni U, et al. A standardised, generic, validated approach to stratify the magnitude of clinical benefit that can be anticipated from anti-cancer therapies: the European Society for Medical Oncology Magnitude of Clinical Benefit Scale (ESMO-MCBS). Ann Oncol. 2015;26(8):1547-1573. doi:10.1093/ annonc/mdv249

38. NICE. Methods, processes and topic selection for health technology evaluation: proposals for change; 2021. Available from: https://www. nice.org.uk/about/what-we-do/our-programmes/nice-guidance/chtemethods-and-processes-consultation. Accessed 14 September, 2021.

39. Cook JP, Golec JH, Vernon JA, Pink GH. Real option value and path dependence in oncology innovation. Int J Econ Bus. 2011;18 (2):225-238. doi:10.1080/13571516.2011.584428
40. Fornaro G, Federici C, Rognoni C, Ciani O. Broadening the concept of value: a scoping review on the option value of medical technologies. Value Health. 2021;24:1045-1058. doi:10.1016/j. jval.2020.12.018

41. FDA in brief: FDA oncologic drugs advisory committee to review status of six indications granted accelerated approval; 2021. Available from: https://www.fda.gov/news-events/fda-brief/fda-brief-fdaoncologic-drugs-advisory-committee-review-status-six-indicationsgranted-accelerated\#: : text $=$ Since $\% 20$ the $\% 20$ inception $\% 20$ of $\%$ 20the,oncology $\% 20$ indications $\% 20$ have $\% 20$ been $\% 20$ withdrawn. Accessed June 15, 2021.

42. Beaver JA, Pazdur R. "Dangling" accelerated approvals in oncology. N Engl J Med. 2021;384(18):e68. doi:10.1056/NEJMp2104846

43. Rupp T, Zuckerman D. Quality of life, overall survival, and costs of cancer drugs approved based on surrogate endpoints. JAMA Intern Med. 2017;177(2):276-277. doi:10.1001/jamainternmed.2016.7761

44. Havrilesky LJ, Lim S, Ehrisman JA, et al. Patient preferences for maintenance PARP inhibitor therapy in ovarian cancer treatment. Gynecol Oncol. 2020;156(3):561-567. doi:10.1016/j.ygyno.2020.01.026

45. DiMasi JA, Grabowski HG, Hansen RW. Innovation in the pharmaceutical industry: new estimates of R\&D costs. $J$ Health Econ. 2016;47:20-33. doi:10.1016/j.jhealeco.2016.01.012

46. Council Directive 89/105/EEC of 21 December 1988 relating to the transparency of measures regulating the prices of medicinal products for human use and their inclusion in the scope of national health insurance systems. Available from: https://eur-lex.europa.eu/legal-content/EN/ TXT/?uri=CELEX\%3A31989L0105. Accessed March 16, 2021.

47. Temple R. A regulatory authority's opinion about surrogate endpoints. In: Nirnnio W, Tucker G, editors. Clinical Measurements in Drug Evaluation. John Wiley \& Sons Ltd; 1995:3-22.

48. Kirkham JJ, Davis K, Altman DG, et al. Core outcome Set-STAndards for development: the COS-STAD recommendations. PLoS Med. 2017;14(11):e1002447. doi:10.1371/journal. pmed. 1002447

49. Naci H, Davis C, Savović J, et al. Design characteristics, risk of bias, and reporting of randomised controlled trials supporting approvals of cancer drugs by European Medicines Agency, 2014-16: cross sectional analysis. BMJ. 2019;366:15221. doi:10.1136/bmj.15221

50. Hilal T, Gonzalez-Velez M, Prasad V. Limitations in clinical trials leading to anticancer drug approvals by the US Food and Drug Administration. JAMA Intern Med. 2020;180(8):1108-1115. doi:10.1001/jamainternmed.2020.2250

51. Fürstenau M, De Silva N, Eichhorst B, Hallek M. Minimal residual disease assessment in CLL: ready for use in clinical routine? HemaSphere. 2019;3(5):e287. doi:10.1097/HS9.0000000000000287

52. EMA. Guideline on the use of minimal residue disease as an endpoint in chronic lymphocytic leukaemia studies; 2014. Available from: https://www.ema.europa.eu/en/documents/scientific-guideline/guide line-use-minimal-residue-disease-endpoint-chronic-lymphocyticleukaemia-studies_en.pdf. Accessed November 11, 2020.

53. Burris H, Storniolo AM. Assessing clinical benefit in the treatment of pancreas cancer: gemcitabine compared to 5-fluorouracil. Eur J Cancer. 1997;33(Suppl 1):S18-22. doi:10.1016/S0959-8049(96)00324-3

54. Korn EL, Freidlin B, Mooney M. Stopping or reporting early for positive results in randomized clinical trials: the National Cancer Institute Cooperative Group experience from 1990 to 2005. J Clin Oncol. 2009;27(10):1712-1721. doi:10.1200/JCO.2008.19.5339

55. AstraZeneca Press Release. Tagrisso Phase III ADAURA trial will be unblinded early after overwhelming efficacy in the adjuvant treatment of patients with EGFR-mutated lung cancer; 2020. Available from: https://www.astrazeneca.com/media-centre/press-releases/2020/ tagrisso-phase-iii-adaura-trial-will-be-unblinded-early-afteroverwhelming-efficacy-in-The-adjuvant-treatment-of-patients-withegfr-mutated-lung-cancer.html. Accessed January 11, 2020. 
56. Ishak KJ, Proskorovsky I, Korytowsky B, Sandin R, Faivre S, Valle J. Methods for adjusting for bias due to crossover in oncology trials. PharmacoEconomics. 2014;32(6):533-546. doi:10.1007/s40273-0140145-y

57. Latimer NR, Abrams KR, Lambert PC, Morden JP, Crowther MJ. Assessing methods for dealing with treatment switching in clinical trials: a follow-up simulation study. Stat Methods Med Res. 2018;27 (3):765-784. doi:10.1177/0962280216642264

58. Poveda A, Floquet A, Ledermann JA, et al. Olaparib tablets as maintenance therapy in patients with platinum-sensitive relapsed ovarian cancer and a BRCA1/2 mutation (SOLO2/ENGOT-Ov21): a final analysis of a double-blind, randomised, placebo-controlled, phase 3 trial. Lancet Oncol. 2021;22:620-631. doi:10.1016/S14702045(21)00073-5

59. de Claro RA, McGinn K, Kwitkowski V, et al. U.S. Food and Drug Administration approval summary: brentuximab vedotin for the treatment of relapsed Hodgkin lymphoma or relapsed systemic anaplastic large-cell lymphoma. Clin Cancer Res. 2012;18(21):5845-5849. doi:10.1158/1078-0432.CCR-12-1803

60. Capocaccia R, Gatta G, Dal Maso L. Life expectancy of colon, breast, and testicular cancer patients: an analysis of US-SEER population-based data. Ann Oncol. 2015;26(6):1263-1268. doi:10.1093/annonc/mdv131

61. Lin DY, Fischl MA, Schoenfeld DA. Evaluating the role of CD4-lymphocyte counts as surrogate endpoints in human immunodeficiency virus clinical trials. Stat Med. 1993;12(9):835-842. doi:10.1002/sim.4780120904

62. Nakagawa F, May M, Phillips A. Life expectancy living with HIV: recent estimates and future implications. Curr Opin Infect Dis. 2013;26(1):17-25. doi:10.1097/QCO.0b013e32835ba6b1

63. Lazarus JV, Safreed-Harmon K, Barton SE, et al. Beyond viral suppression of HIV - the new quality of life frontier. BMC Med. 2016;14(1):94. doi:10.1186/s12916-016-0640-4

64. Hullsiek KH, Grund B. Considerations for endpoint selection when designing HIV clinical trials. Curr Infect Dis Rep. 2012;14 (1):110-118. doi:10.1007/s11908-011-0231-7

65. Wilson ECF. A practical guide to value of information analysis. PharmacoEconomics. 2015;33(2):105-121. doi:10.1007/s40273014-0219-x

66. Buyse M, Saad ED, Peron J, et al. The Net Benefit of a treatment should take the correlation between benefits and harms into account. J Clin Epidemiol. 2021;137:148-158. doi:10.1016/j.jclinepi.2021.03.018

67. Muller V, Nabieva N, Haberle L, et al. Impact of disease progression on health-related quality of life in patients with metastatic breast cancer in the PRAEGNANT breast cancer registry. Breast. 2018;37:154-160. doi:10.1016/j.breast.2017.08.008

68. NICE. Pertuzumab for the neoadjuvant treatment of HER2-positive breast cancer -technology appraisal guidance [TA424]: final appraisal document; 2016. Available from: https://www.nice.org.uk/guidance/ ta424/documents/final-appraisal-determination-document. Accessed March 4, 2021.

69. Harbeck N, Schneeweiss A, Thuss-Patience P, et al. Neoadjuvant and adjuvant end-points in health technology assessment in oncology. Eur J Cancer. 2021;147:40-50. doi:10.1016/j.ejca.2021.01.006

70. Marschner N, Zacharias S, Lordick F, et al. Association of disease progression with health-related quality of life among adults with breast, lung, pancreatic, and colorectal cancer. JAMA Netw Open. 2020;3(3):e200643. doi:10.1001/jamanetworkopen.2020.0643

71. Wu Y-L, Tsuboi M, He J, et al. Osimertinib in resected EGFRmutated non-small-cell lung cancer. $N$ Engl J Med. 2020;383 (18):1711-1723. doi:10.1056/NEJMoa2027071

72. Bottomley A, Pe M, Sloan J, et al. Analysing data from patient-reported outcome and quality of life endpoints for cancer clinical trials: a start in setting international standards. Lancet Oncol. 2016;17(11):e510-e514. doi:10.1016/S1470-2045(16)30510-1
73. Wilson MK, Mercieca-Bebber R, Friedlander M. A practical guide to understanding, using and including patient reported outcomes in clinical trials in ovarian cancer. J Gynecol Oncol. 2018;29(5):e81. doi:10.3802/jgo.2018.29.e81

74. Joly F, Hilpert F, Okamoto A, Stuart G, Ochiai K, Friedlander M. Fifth Ovarian Cancer Consensus Conference of the Gynecologic Cancer InterGroup: recommendations on incorporating patient-reported outcomes in clinical trials in epithelial ovarian cancer. Eur J Cancer. 2017;78:133-138. doi:10.1016/j. ejca.2017.03.019

75. Gnanasakthy A, Barrett A, Evans E, D'Alessio D, Romano C. A review of patient-reported outcomes labeling for oncology drugs approved by the FDA and the EMA (2012-2016). Value Health. 2019;22(2):203-209. doi:10.1016/j.jval.2018.09.2842

76. Wood R, Taylor-Stokes G, Smith F, Chaib C. The humanistic burden of advanced non-small cell lung cancer (NSCLC) in Europe: a real-world survey linking patient clinical factors to patient and caregiver burden. Qual Life Res. 2019;28(7):1849-1861. doi:10.1007/ s11136-019-02152-6

77. Friends of Cancer Research. Broadening the definition of tolerability in cancer clinical trials to better measure the patient experience; 2018. Available from: https://www.focr.org/sites/default/files/ Comparative\%20Tolerability\%20Whitepaper_FINAL.pdf. Accessed January 12, 2021.

78. Havrilesky LJ, Alvarez Secord A, Ehrisman JA, et al. Patient preferences in advanced or recurrent ovarian cancer. Cancer. 2014;120 (23):3651-3659. doi:10.1002/cncr.28940

79. Gyawali B, Hey SP, Kesselheim AS. Evaluating the evidence behind the surrogate measures included in the FDA's table of surrogate endpoints as supporting approval of cancer drugs. EClinicalMedicine. 2020;21:100332. doi:10.1016/j.eclinm.20 20.100332

80. National Cancer Institute. National Cancer Institute dictionary of cancer terms. Available from: https://www.cancer.gov/publications/ dictionaries/cancer-terms. Accessed May 14, 2020.

81. Xie W, Regan MM, Buyse M, et al. Metastasis-free survival is a strong surrogate of overall survival in localized prostate cancer. J Clin Oncol. 2017;35(27):3097-3104. doi:10.1200/JCO.2017.73.9987

82. Grigore B, Ciani O, Dams F, et al. Surrogate endpoints in health technology assessment: an international review of methodological guidelines. PharmacoEconomics. 2020;38(10):1055-1070. doi:10.1007/s40273-020-00935-1

83. Powers JH, Patrick DL, Walton MK, et al. Clinician-reported outcome assessments of treatment benefit: report of the ISPOR clinical outcome assessment emerging good practices task force. Value Health. 2017;20(1):2-14. doi:10.1016/j.jval.2016.11.005

84. Ciani O, Buyse M, Drummond M, Rasi G, Saad ED, Taylor RS. Time to review the role of surrogate end points in health policy: state of the art and the way forward. Value Health. 2017;20(3):487-495. doi:10.1016/j.jval.2016.10.011

85. Buyse M, Sargent DJ, Grothey A, Matheson A, de Gramont A. Biomarkers and surrogate end points - the challenge of statistical validation. Nat Rev Clin Oncol. 2010;7(6):309-317. doi:10.1038/ nrclinonc. 2010.43

86. Prentice RL. Surrogate endpoints in clinical trials: definition and operational criteria. Stat Med. 1989;8(4):431-440. doi:10.1002/ sim.4780080407

87. Ciani O, Grigore B, Blommestein H, et al. Validity of surrogate endpoints and their impact on coverage recommendations: a retrospective analysis across International Health Technology Assessment Agencies. Med Decis Making. 2021;41:439-452.

88. Ouwens MJNM, Mukhopadhyay P, Zhang Y, Huang M, Latimer N, Briggs A. Estimating lifetime benefits associated with immuno-oncology therapies: challenges and approaches for overall survival extrapolations. PharmacoEconomics. 2019;37 (9):1129-1138. doi:10.1007/s40273-019-00806-4 
89. Bullement A, Meng Y, Cooper M, et al. A review and validation of overall survival extrapolation in health technology assessments of cancer immunotherapy by the National Institute for Health and Care Excellence: how did the initial best estimate compare to trial data subsequently made available? J Med Econ. 2019;22(3):205-214.

90. Tai TA, Latimer NR, Benedict A, Kiss Z, Nikolaou A. Prevalence of immature survival data for anti-cancer drugs presented to the National Institute for Health and Care Excellence and impact on decision making. Value Health. 2021;24(4):505-512. doi:10.1016/j. jval.2020.10.016

91. NICE. Broader types of data to be used in development of NICE guidance; 2020. Available from: https:/www.nice.org.uk/news/arti cle/broader-types-of-data-to-be-used-in-development-of-niceguidance. Accessed November 11, 2020.

92. NICE Decision Support Unit. The use of real world data for the estimation of treatment effects in NICE decision making; 2016. Available from: http:/nicedsu.org.uk/wp-content/uploads/2018/05/ RWD-DSU-REPORT-Updated-DECEMBER-2016.pdf. Accessed November 11, 2020.

93. Lakdawalla DN, Shafrin J, Hou N, et al. Predicting real-world effectiveness of cancer therapies using overall survival and progression-free survival from clinical trials: empirical evidence for the ASCO value framework. Value Health. 2017;20(7):866-875. doi:10.1016/j.jval.2017.04.003
94. Lux MP, Nabieva N, Hartkopf AD, et al. Therapy landscape in patients with metastatic HER2-positive breast cancer: data from the PRAEGNANT real-world breast cancer registry. Cancers (Basel). 2018;11(1):10. doi:10.3390/cancers 11010010

95. Schneeweiss A, Ettl J, Lüftner D, et al. Initial experience with CDK4/ 6 inhibitor-based therapies compared to antihormone monotherapies in routine clinical use in patients with hormone receptor positive, HER2 negative breast cancer - Data from the PRAEGNANT research network for the first 2 years of drug availability in Germany. Breast. 2020;54:88-95.

96. Annemans L, Makady A. TRUST4RD: tool for reducing uncertainties in the evidence generation for specialised treatments for rare diseases. Orphanet J Rare Dis. 2020;15(1):127. doi:10.1186/ s13023-020-01370-3

97. DeMichele A, Cristofanilli M, Brufsky A, et al. Abstract P1-19-02: overall survival for first-line palbociclib plus letrozole vs letrozole alone for HR+/HER2- metastatic breast cancer patients in US real-world clinical practice. Cancer Res. 2020;80(4Supplement): P1-19-02-P11-19-02.

\section{Publish your work in this journal}

Cancer Management and Research is an international, peer-reviewed open access journal focusing on cancer research and the optimal use of preventative and integrated treatment interventions to achieve improved outcomes, enhanced survival and quality of life for the cancer patient.
The manuscript management system is completely online and includes a very quick and fair peer-review system, which is all easy to use. Visit http://www.dovepress.com/testimonials.php to read real quotes from published authors. 